\title{
Editorial
}

\section{Qualitative Analysis of Differential, Difference Equations, and Dynamic Equations on Time Scales}

\author{
Tongxing Li, ${ }^{1}$ Josef Diblík, ${ }^{2,3}$ Alexander Domoshnitsky, ${ }^{4}$ Yuriy V. Rogovchenko, ${ }^{5}$ \\ Felix Sadyrbaev, ${ }^{6}$ and Qi-Ru Wang ${ }^{7}$ \\ ${ }^{1}$ School of Informatics, Linyi University, Linyi, Shandong 276005, China \\ ${ }^{2}$ Department of Mathematics and Descriptive Geometry, Faculty of Civil Engineering, Brno University of Technology, \\ 60200 Brno, Czech Republic \\ ${ }^{3}$ Department of Mathematics, Faculty of Electrical Engineering, Brno University of Technology, 61600 Brno, Czech Republic \\ ${ }^{4}$ Department of Mathematics and Computer Sciences, Ariel University, 40700 Ariel, Israel \\ ${ }^{5}$ Department of Mathematical Sciences, University of Agder, P.O. Box 422, 4604 Kristiansand, Norway \\ ${ }^{6}$ Institute of Mathematics and Computer Science, University of Latvia, Rainis Boulevard 29, Riga LV-1459, Latvia \\ ${ }^{7}$ School of Mathematics and Computational Science, Sun Yat-sen University, Guangzhou 510275, China \\ Correspondence should be addressed to Tongxing Li; litongx2007@163.com
}

Received 4 November 2014; Accepted 4 November 2014

Copyright (C) 2015 Tongxing Li et al. This is an open access article distributed under the Creative Commons Attribution License, which permits unrestricted use, distribution, and reproduction in any medium, provided the original work is properly cited.

It is our pleasure to present this special issue. Differential, difference, and dynamic equations on time scales are often used for modeling various problems arising in the engineering and natural sciences. Therefore, analysis of qualitative properties of solutions to such equations is crucial for applications. It is important to develop new efficient methods, as well as to modify and refine well-known techniques adjusting them for the analysis of new classes of problems.

In the call for papers prepared by the Guest Editors, we encouraged submission of state-of-the-art contributions on a wide spectrum of topics including asymptotic behavior of solutions, oscillation and nonoscillation, solvability of boundary value problems, existence of periodic and almost periodic solutions, stability properties of solutions, and applications to real world phenomena. This invitation was warmly welcomed by the mathematical community; more than sixty manuscripts addressing important problems in related areas were submitted to the Editorial Office and went through a thorough peer refereeing process. Thirty-eight research articles and two review articles reflecting modern trends and advances in functional differential, difference, and dynamic equations on time scales have been selected for this special issue.
In the contribution by Y. Zhang et al., an adaptive scheme that can be used to analyze an open-circuit voltage of a battery is presented. H. Xi et al. study global behavior of a class of difference equations. In the paper by R. Guo, an improved average dwell time method is utilized to establish a number of stability results for a class of switched nonlinear systems. L. Gao et al. study a class of thirdorder nonlinear delay dynamic equations on time scales, whereas Q. Zhang et al. deal with a class of third-order nonlinear functional differential equations. Both papers establish sufficient conditions which ensure that every solution of a given equation is either oscillatory or converges to zero. For a class of two-dimensional nonlinear dynamic systems on time scales with a forced term, X. Zhang and S. Zhu obtain sufficient conditions for all solutions to be oscillatory. D. Cai et al. introduce a generalized Solow-Swan model for the analysis of the exogenous impact of population, saving rate, technological change, and labor participation rate on the economic growth. Y.-C. Qiu and Q.-R. Wang establish new oscillation results for a class of second-order nonlinear damped dynamic equations of a more general form. Y. Sun and T. S. Hassan present new oscillation criteria for a class of second-order nonlinear dynamic equations. 
In the paper by F. Wang, a comparison theorem for a fully nonlinear path-dependent parabolic partial differential equation is presented and some applications are considered. Z. Jiang et al. prove that the set of skew-circulants with complex entries has an idempotent basis. On this basis, a skewcyclic group of automorphisms and functional equations on the skew-circulant algebra is introduced. Using the inverse factorization of polynomial of degree $n, \mathrm{~J}$. Li et al. express determinants of circulant and left circulant matrix involving Tribonacci numbers or generalized Lucas numbers in terms of Tribonacci numbers and generalized Lucas numbers. Q. He et al. investigate periodic nature of positive solutions of a class of fuzzy max-difference equations. Q. Li et al. discuss asymptotic properties of a class of third-order nonlinear neutral functional differential equations. In the paper by $\mathrm{X}$. Jiang and $\mathrm{K}$. Hong, the determinant problems for RSFPLR circulant matrices and RSLPFL circulant matrices involving Perrin, Padovan, Tribonacci, and generalized Lucas numbers are considered. In the paper by $\mathrm{X}$. Fu et al., an energy management strategy for a plug-in hybrid electric vehicle based on the driving cycle model and dynamic programming algorithm is proposed.

$Z$. Jiang introduces a level- $k$ scaled factor circulant matrix over an arbitrary field and discusses its algebraic properties. S. Liu and Q. Zhang establish oscillation criteria of Gracetype for a class of second-order nonlinear dynamic equations with damping. Z. Jiang explores basic properties of FLS $R$ factor block circulant (retrocirculant) matrices. Z. Jiang et al. consider circulant type matrices, including the circulant and left circulant and $g$-circulant matrices with the sum and product of Fibonacci and Lucas numbers. T. Xu et al. obtain explicit expressions for determinants of the RFP $r \mathrm{~L} r \mathrm{R}$ circulant matrices and RLPrFrL circulant matrices involving Fibonacci, Lucas, Pell, and Pell-Lucas numbers. In the paper by F. Yang and Q. Li, an improved cross entropy algorithm is proposed to solve the charge planning problem with orders in each charge. Using the nonlinear alternative of LeraySchauder type and Banach fixed-point theorem, W.-X. Zhou and $\mathrm{H}$. Liu establish existence and uniqueness of solution for a system of fractional differential equations. In the contribution by Q. H. Alqifiary and S.-M. Jung, the Gronwall inequality is used to prove the Hyers-Ulam stability of a class of secondorder differential equations.

P. Hasil et al. demonstrate that existence of the mean values of coefficients is sufficient for second-order half-linear Euler-type differential equations to be conditionally oscillatory. An oscillation constant is determined explicitly even for equations with coefficients of variable sign. Using the method of coincidence degree, Y. Wang and M. Han construct a suitable Lyapunov functional to prove the existence of antiperiodic solutions of impulsive Cohen-Grossberg neural networks with delays on time scales and to investigate global exponential stability. The paper by S. R. Grace and M. A. ElBeltagy deals with the oscillatory behavior of forced secondorder integrodynamic equations on time scales. The results are new for the continuous and discrete cases and can be applied to Volterra integral equations on time scales. Two ellipsoidal ultimate boundary regions for a special threedimensional chaotic system are suggested by J. Wang et al.
C. Wu et al. study two discrete predator-prey models in patchy environment, one without dispersal corridors and one with dispersal corridors. In the paper by $\mathrm{H}$. Chen et al., a new method called a homoclinic (heteroclinic) breather limit method is proposed to find rogue wave solutions to nonlinear evolution equations.

Using the generalized Riccati transformation and inequality technique, Q. Zhang and S. Liu establish oscillation criteria of Philos-type for second-order half-linear neutral delay dynamic equations with damping on time scales. Z.-L. Han et al. present several oscillation results for a class of even-order neutral delay differential equations with mixed nonlinearities. M. Dobkevich et al. present results on classification of solutions to boundary value problems for a class of second-order nonlinear differential equations. In this framework, existence and multiplicity results for different types of solutions are stated. Employing Lyapunov functions, Razumikhin method, and comparison principle, P. Wang et al. investigate relative integral stability of two differential systems with "maxima" in terms of two measures. In the contribution by A. Domoshnitsky et al., sufficient conditions for nonnegativity of a Cauchy matrix for a delay system are obtained without assuming nonpositivity of nondiagonal coefficients and a necessary condition for nonnegativity of a Cauchy matrix is suggested. Using results on nonnegativity of a Cauchy matrix, necessary and sufficient conditions for the exponential stability of a delay system are derived.

H. Gu and T. An use a variant of the fountain theorem to study existence of multiple solutions for a class of superquadratic fourth-order elliptic problems with Navier boundary value conditions. X.-M. Zheng et al. investigate fixed points of solutions to a class of $q$-difference equations. In the paper by Y. Zhang et al., the dynamics of a disease in a population of migratory birds modeled by a nonautonomous system with a Hassell-Varley type functional response and the saturation incidence rate is studied. Sufficient conditions are obtained for the permanence and extinction of the disease. Global attractivity of the model is discussed by constructing a Lyapunov function. C. Jiang et al. propose a new fractional-order chaotic complex system and study its dynamic properties including symmetry, equilibria and their stability, and chaotic attractors. The contribution by L. Caso et al. that reviews results on the unique solvability of the Dirichlet problem for second-order linear elliptic partial differential equations in nondivergence form with singular data in weighted Sobolev spaces closes this special issue.

The editors hope that this collection of papers will attract interest of researchers working in related areas and will stimulate further progress in the qualitative theory of differential, difference, and dynamic equations on time scales.

\section{Acknowledgments}

The editorial team would like to express gratitude to the authors for their contributions and to thank all reviewers whose invaluable expertise and helpful recommendations assured high quality of papers selected for this special issue. Finally, the Lead Guest Editor Tongxing Li would like to 
thank Guest Editors Josef Diblík, Alexander Domoshnitsky, Yuriy V. Rogovchenko, Felix Sadyrbaev, and Qi-Ru Wang for their unfailing support with editorial work that ensured timely preparation of this special edition.

Tongxing $L i$

Josef Diblik

Alexander Domoshnitsky

Yuriy V. Rogovchenko

Felix Sadyrbaev

Qi-Ru Wang 


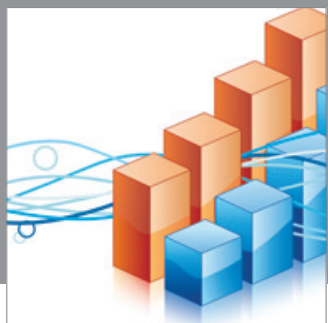

Advances in

Operations Research

mansans

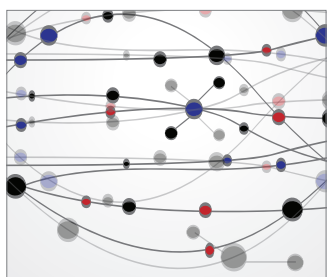

The Scientific World Journal
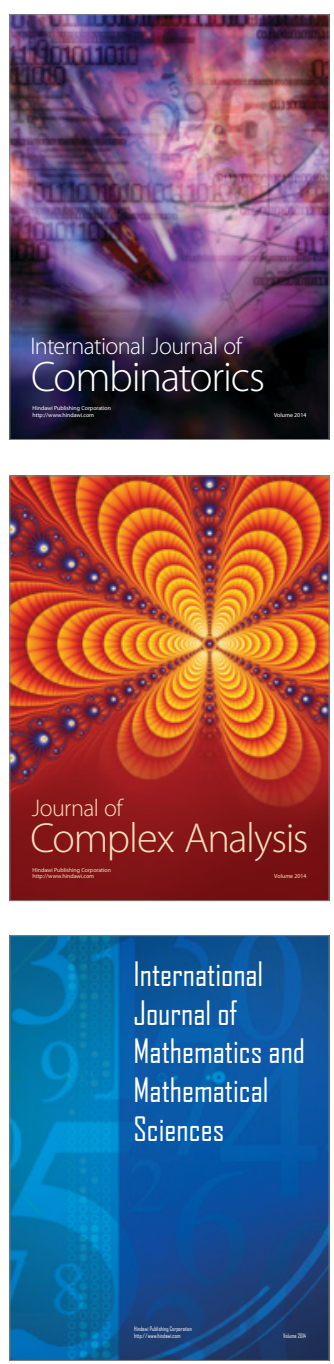
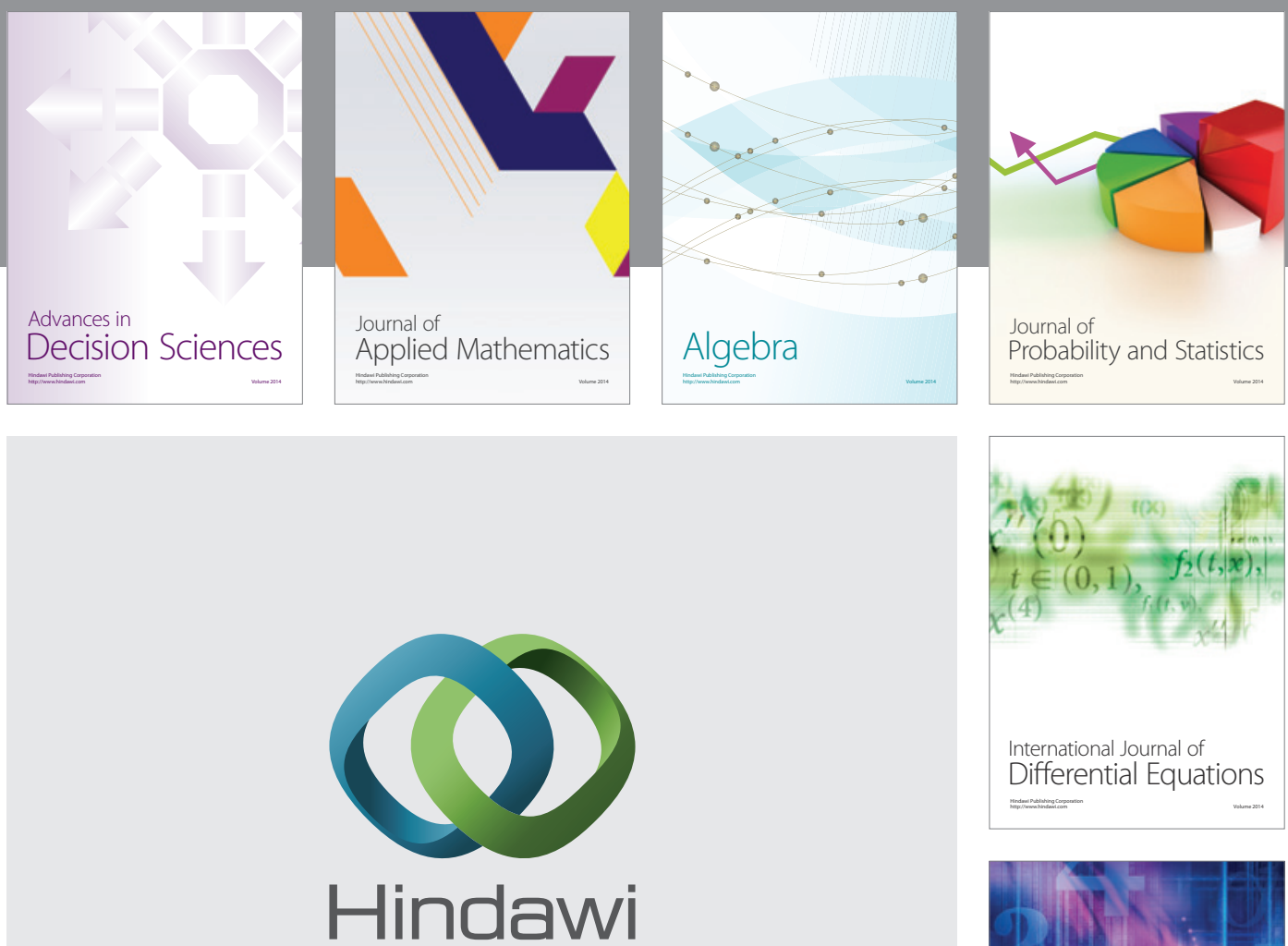

Submit your manuscripts at http://www.hindawi.com
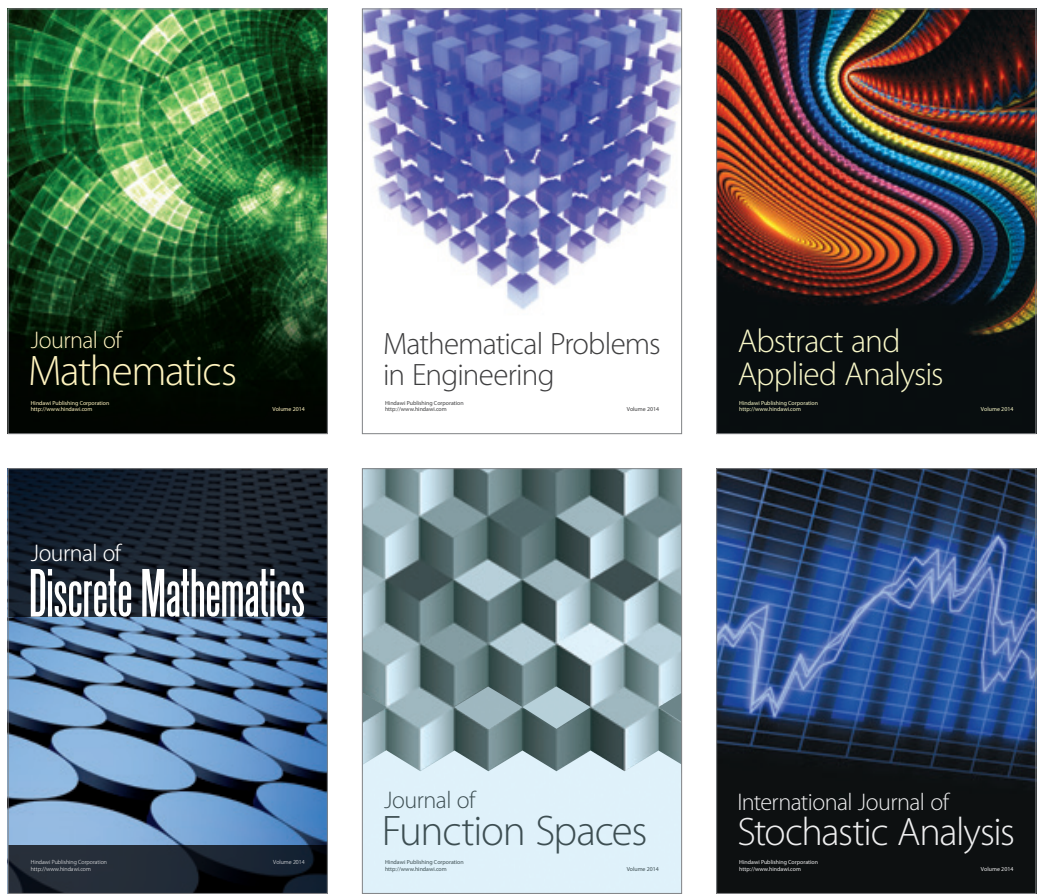

Journal of

Function Spaces

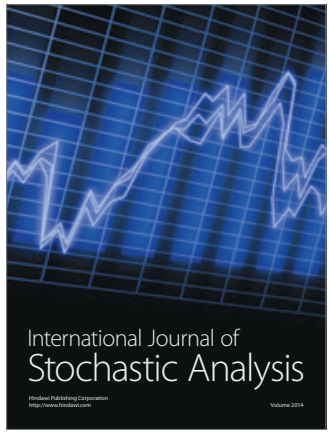

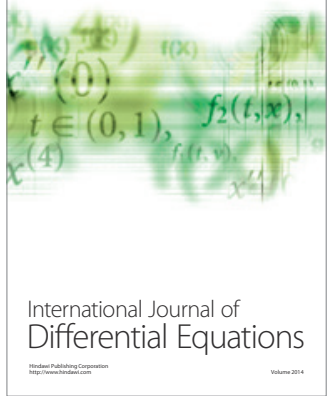
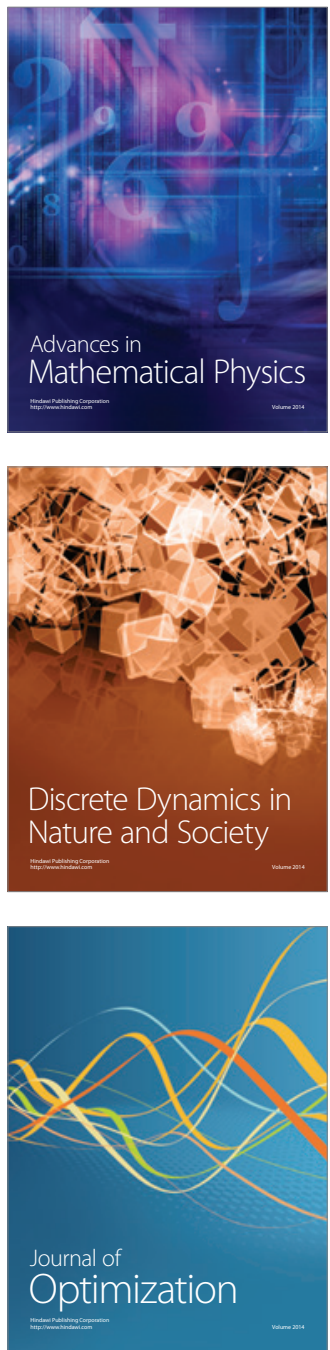